\title{
Diversité des insectes floricoles et son impact sur les rendements fruitiers et grainiers de Arachis hypogaea L. (Fabaceae) à Dang (Ngaoundéré-Cameroun)
}

\author{
Fernand-Nestor TCHUENGUEM FOHOUO ${ }^{1}$, Bernice Mireille KINGHA TEKOMBO ${ }^{1 *}$ et \\ Dorothea BRÜCKNER ${ }^{2}$ \\ ${ }^{1}$ Université de Ngaoundéré, Faculté des Sciences, Département des Sciences Biologiques, \\ B.P. 454 Ngaoundéré, Cameroun. \\ ${ }^{2}$ Forschungsstelle für Bienenkunde, Universität Bremen, FB2, Postfach 3304 40, 28334 Bremen, Allemagne. \\ *Auteur correspondant, E-mail: bernice2905@yahoo.fr, Tel : (237) 991462 21/74 283182
}

\section{RESUME}

De juin à septembre, en 2009 et 2010 respectivement, les fleurs de Arachis hypogaea ont été observées en vue de l'étude de l'activité des insectes anthophiles et de la détermination de l'impact de ceux-ci sur les rendements fruitiers et grainiers de cette plante. Deux traitements ont été déterminés à partir du marquage de six billons différenciés par la présence ou l'absence de protection des billons vis-à-vis des insectes. L'activité de butinage et de pollinisation des insectes a été étudiée. Le taux de fructification, le pourcentage de graines normales et le nombre moyen de graines par gousse ont été évalués. Les résultats montrent que 17 et 12 espèces d'insectes ont visité les fleurs d'arachide respectivement en 2009 et 2010. Lasioglossum sp. 1 et Ceratina sp. 2 sont les espèces les plus fréquemment observées en 2009 et 2010 respectivement. En comparant les rendements des deux traitements, il est apparu que les insectes anthophiles ont augmenté le taux de fructification de $24,83 \%$ et $54,47 \%$, le pourcentage de graines normales de $28,65 \%$ et $24,89 \%$, le nombre moyen de graines par gousse de $15,03 \%$ et $14,90 \%$ respectivement en 2009 et 2010 . Ainsi, les traitements aux pesticides chimiques sont à éviter pendant la période de floraison.

(C) 2014 International Formulae Group. All rights reserved.

Mots clés: Insectes anthophiles, Arachis hypogaea, butinage, pollen, pollinisation, rendements.

\section{INTRODUCTION}

Au Cameroun, depuis plus de vingt quatre ans, les recherches sont effectuées sur les relations plantes - insectes pollinisateurs, notamment dans les régions du Centre (Tchuenguem Fohouo, 1993; Pando et al., 2011a, 2011b), de l'Ouest (Dongock et al., 2004), de l'Adamoua (Tchuenguem Fohouo et al., 2009a, 2009b; Kingha et al., 2012; Fameni et al., 2012) et de l'Extrême-Nord (Douka et Tchuenguem Fohouo, 2013; Dounia et Tchuenguem Fohouo, 2013). Malgré ces travaux, les informations manquent sur les relations entre plusieurs plantes poussant au Cameroun et leurs insectes floricoles. Pourtant il est bien connu que les insectes anthophiles augmentent habituellement les rendements en fruits et/ou en grains de plusieurs espèces végétales, via la pollinisation des fleurs au cours de leur activité de butinage (Tchuenguem Fohouo, 1993; Kingha et al., 2012; Fameni et al., 2012; Pando et al., 2011a, 2011b; Douka et Tchuenguem Fohouo, 2013; Dounia et Tchuenguem Fohouo, 2013). 
Arachis hypogaea est une Fabaceae originaire du Brésil (Vallardir, 1964). C'est une plante cultivée dans tous les pays tropicaux, subtropicaux et même dans certains pays d'Europe (Pouvreau, 1984). Les fleurs sont jaunes et produisent du pollen qui attire les insectes. Elles ne secrètent pas de nectar (Pouvreau, 1984). L'arachide est une légumineuse ayant une grande importance nutritionnelle et économique dans toute la zone intertropicale (Kalfaoui, 1988). Cultivée sur 26,4 millions d'hectares à l'échelle mondiale, la production totale est de 37,1 millions de tonnes pour une productivité moyenne de 1,4 tonne à l'hectare (FAO, 2003). Elle est fortement cultivée à l'Ouest du Cameroun où son rendement moyen demeure faible (Praquin et Tardieu, 1976). Dans ce pays, la demande en graines d'arachide est estimée à 609312 tonnes (DSCE, 2009) et la production disponible n'est que de 484199 tonnes (MINADER/DESA, 2010). De plus, très peu de données ont été publiées sur ses relations avec les insectes floricoles. $\mathrm{Au}$ Malawi, Apis mellifica adansonii, Nomia spp. et Megachile spp. sont les visiteurs les plus communs (Gibbons et Tattersfield, 1969); en Israël, Carmin (Free, 1970) a noté que Ceratina bispinosa est le principal insecte floricole d'arachide. Rashad et al. (1978) ont signalé qu'en Egypte, les insectes pollinisateurs augmentent les rendements de l'arachide d'environ 46\%. A Yaoundé, au Cameroun, Tchuenguem Fohouo (1993) a montré que les insectes anthophiles augmentent les rendements en graines et en des gousses de l'arachide de $29,59 \%$ et de $28,13 \%$ respectivement, bien que la diversité et l'abondance des insectes anthophiles d'une plante peuvent varier avec les régions (Roubik, 2000).

L'objectif général de cette recherche est de contribuer à la connaissance des relations existant entre $A$. hypogaea et ses insectes anthophiles en vue de leur exploitation optimale au Cameroun.

Les objectifs spécifiques incluent: la détermination de la diversité de l'entomofaune anthophile de A. hypogaea, l'étude de l'activité des insectes sur les fleurs de cette
Fabaceae, l'estimation de la valeur apicole de cette plante, l'évaluation de l'impact des insectes floricoles sur la pollinisation et les rendements en gousses et en graines de cette légumineuse.

\section{MATERIEL ET METHODES \\ Site, champ expérimental et matériel biologique \\ Les travaux ont été réalisés de juin à} septembre 2009, puis de juin à septembre 2010 à Dang (latitude: 7²5.372' N, longitude: 13³2.566' E, altitude: $1092 \mathrm{~m}$ ), village de Ngaoundéré dans la région de l'Adamaoua au Cameroun. Cette région appartient à la zone écologique dite des hautes savanes guinéennes (Tchuenguem Fohouo et al., 2007). Le climat est caractérisé par deux saisons: une saison des pluies (avril à octobre) et une saison sèche (novembre à mars). La pluviométrie annuelle est d'environ $1500 \mathrm{~mm}$. La température moyenne annuelle est de 22 ${ }^{\circ} \mathrm{C}$. L'humidité relative moyenne annuelle est de 70\% (Tchuenguem Fohouo et al., 2007). Le champ expérimental était une parcelle de $230 \mathrm{~m}^{2}$ où les graines de A. hypogaea de la variété 55-437 RMP-91 (Figure 1) ont été semées. Sur l'ensemble du site d'étude, le nombre de colonies de Apis mellifera adansonii variait de 31 à 48 entre juin 2009 et octobre 2010 hors mis les insectes naturellement présents dans l'environnement. La végétation était représentée par d'autres plantes cultivées puis quelques espèces naturelles de la savane et des galeries forestières.

\section{Méthodes \\ Préparation du champ expérimental, semis et entretien de la culture}

Du 21 avril au 08 mai 2009, puis du 21 avril au 08 mai 2010, les parcelles expérimentales ont été défrichées, labourées et 18 billons de $3 \mathrm{~m}$ de long, $1 \mathrm{~m}$ de large et 10 cm de hauteur ont été formés. Le 29 mai 2009 et le 23 juin 2010, le semi a été fait en ligne, à raison de 5 lignes par billon et une graine par poquet. Les espacements sur les lignes et entre les lignes étaient de $15 \mathrm{~cm}$. De la germination (survenue le 05 juin 2009 et le 30 juin 2010) à 
l'apparition des premières fleurs (le 29 juin 2009 et le 24 juillet 2010), le sarclage a été régulièrement effectué à la houe toutes les deux semaines. Durant la période de floraison jusqu'à la maturation des gousses, le désherbage a été fait à la main.

\section{Détermination du système de reproduction}

Le 25 juin 2009, 1200 plantes de $A$. hypogaea ont été isolées à raison de 100 par billon et deux traitements constitués: traitement 1 (600 plantes dont les fleurs ont été laissées en libre pollinisation et sur lesquelles aucune capture d'insectes n'a été faite) et traitement 2 (600 plantes dont les fleurs ont été protégées des insectes à l'aide des cages grillagées à mailles fines de $1 \mathrm{~mm}^{2}$ ). Le 20 juillet 2010, l'opération a été répétée.

A la récolte, le nombre de gousses formées a été compté dans chacun des traitements 1 et 2 . Pour chaque traitement, l'indice de fructification (Ifr) a été calculé selon la formule ci-après:

Ifr $=F_{2} / F_{1}$, où $F_{2}$ est le nombre de gousses formées et $F_{1}$ le nombre de fleurs viables initialement portées (Tchuenguem Fohouo et al., 2001).

Pour chaque saison d'observation, la différence entre les indices de fructification des deux traitements a permis de calculer les taux d'allogamie (TC) et d'autogamie $(T A)$ au sens large, selon les formules ci-après (Demarly, 1977):

$T C=\left\{\left[\left(I f r_{1}-I f r_{2}\right) / I f r_{1}\right] \times 100\right\}$ où $I f r_{1}$ et $I f r_{2}$ sont respectivement les indices de fructification dans le traitement libre et dans le traitement protégé ; $T A=[(1-T C)$ x 100].

\section{Etude de l'activité des insectes sur les fleurs}

Sur les fleurs du traitement 1, les observations ont été effectuées tous les jours, du 29 juin au 30 septembre 2009 puis du 24 juillet au 30 septembre 2010, selon six tranches horaires journalières: $6-7 \mathrm{~h}, 8-9 \mathrm{~h}$, $10-11$ h, $12-13 \mathrm{~h}, 14-15$ h et $16-17 \mathrm{~h}$. Nous passions plusieurs fois sur chaque billon du traitement 1 et ceci pendant chacune des tranches horaires ci-dessus. A chaque passage, les différents insectes rencontrés sur les fleurs étaient identifiés et comptés. Les insectes n'ayant pas été marqués, les résultats cumulés ont été exprimés par le nombre de visites
(Tchuenguem Fohouo, 2005). Les données obtenues ont permis de déterminer la fréquence de chaque espèce d'insecte $(F i)$ sur les fleurs de $A$. hypogaea. Pour chaque saison d'étude, $F i=\{[V i / V I] \times 100\}$, avec $V i=$ nombre de visites de l'insecte $i$ sur les fleurs du traitement libre et $V I$ le nombre de visites de tous les insectes sur ces mêmes fleurs (Tchuenguem Fohouo et al., 2001).

Les produits floraux (nectar et/ou pollen) prélevés par les insectes ont été notés pendant les mêmes dates et tranches horaires que pour la fréquence des visites. Un insecte qui enfonce sa trompe ou sa tête dans une fleur est un chercheur de nectar; si à l'aide de ses pattes et de ses mandibules, il gratte les anthères, il s'agit d'un chercheur de pollen (Jean - Prost, 1987).

Les abondances par fleur ont été enregistrées à la suite des comptages directs. Pour l'abondance par 1000 fleurs, les individus de chaque espèce ont été comptés sur un nombre connu de fleurs épanouies ; l'abondance par 1000 fleurs $\left(A_{1000}\right)$ a ensuite été calculée à l'aide de la formule suivante: $A_{1000}=\left[\left(A_{x} / F_{x}\right)\right.$ x 1000$]$, où $F_{x}$ et $A_{x}$ sont respectivement le nombre de fleurs et le nombre d'insectes butineurs effectivement comptés sur les fleurs laissées en libre pollinisation à l'instant $x$ (Tchuenguem Fohouo, 2005). Les données ont été enregistrées pendant les mêmes dates et périodes journalières que pour la fréquence des visites.

La durée des visites par fleur est le temps que met l'abeille pour prélever un produit (pollen et/ou nectar) sur une fleur (Tchuenguem Fohouo, 2005). Elle a été enregistrée aux mêmes dates pendant les tranches horaires journalières suivantes : $7-8$ $\mathrm{h} ; 9-10 \mathrm{~h} ; 11-12 \mathrm{~h} ; 13-14 \mathrm{~h} ; 15-16 \mathrm{~h}$ et 17 - $18 \mathrm{~h}$, à l'aide d'un chronomètre.

La vitesse de butinage selon JacobRemacle (1989) correspond au nombre de fleurs visitées par minute. Ces données ont été enregistrées tous les jours pendant 6 tranches horaires: $7-8 \mathrm{~h} ; 9-10 \mathrm{~h} ; 11-12 \mathrm{~h} ; 13-14$ $\mathrm{h} ; 15-16 \mathrm{~h}$ et $17-18 \mathrm{~h}$, à l'aide d'un chronomètre. 
L'influence de la faune et de la flore avoisinante a été systématiquement enregistrée lors du chronométrage de la durée des visites par fleur.

La température et l'hygrométrie de la station d'étude ont été enregistrées toutes les 30 minutes, à l'aide d'un thermohygromètre portable de marque techno WS-7018 installé à l'ombre, tout au long de la période d'observation.

\section{Estimation de la valeur apicole}

Comme pour d'autres plantes (Tchuenguem Fohouo, 2005; Tchuenguem Fohouo et al., 2007), la valeur apicole de $A$. hypogaea a été évaluée à l'aide principalement des données sur son intensité de floraison et l'attractivité du pollen de cette Fabaceae vis-àvis des ouvrières de $A$. m. adansonii.

\section{Evaluation de l'impact des insectes anthophiles sur les rendements}

Elle est basée sur:

- l'impact des insectes anthophiles sur la pollinisation ;

- l'impact de la pollinisation sur la fructification de A. hypogaea;

- la comparaison des rendements fruitiers et grainiers (taux de fructification, nombre moyen de graines par gousse et pourcentage de graines normales) des traitements 1 et 2.

Le pourcentage $\left(P_{i}\right)$ du taux de fructification dû à l'influence des insectes floricoles est calculé à l'aide de la formule ci après (Tchuenguem Fohouo et al., 2001):

$P_{i}=\left\{\left[\left(F_{1}-F_{2}\right) / F_{1}\right] x 100\right\}$ où $F_{1}$ et $F_{2}$ sont les taux de fructification dans les traitements 1 et 2 respectivement. Pour un traitement, le taux de fructification $=$ (nombre de gousses $/$ nombre de fleurs) $\mathrm{x} 100$.

Le pourcentage $\left(P_{g}\right)$ du nombre de graines par gousse attribuable à l'influence des insectes floricoles est calculé à l'aide de la formule ci - après (Tchuenguem Fohouo et al., 2001):

$P_{g}=\left\{\left[\left(g_{1}-g_{2}\right) / g_{1}\right] \times 100\right\}$ où $g_{1}$ et $g_{2}$ sont les nombres moyens de graines par gousse dans les traitements 1 et 2 respectivement.

Le pourcentage $\left(P_{n}\right)$ de graines normales attribuable à l'influence des insectes floricoles est calculé à l'aide de la formule ci-après (Tchuenguem Fohouo et al., 2001): $P_{n}=\left\{\left(\left[P_{n 1}-P_{n 2}\right) / P_{n 1}\right] \times 100\right\}$ où $P_{n 1}$ et $P_{n 2}$ sont les pourcentages de graines normales dans les traitements 1 et 2 respectivement.

\section{Analyse des données}

Le traitement des données a été fait à l'aide de la statistique descriptive (calcul des moyennes, écart-types et pourcentages), du Coefficient de corrélation $(r)$ pour l'étude des relations entre deux variables, du test $t$ de Student pour la comparaison des moyennes de deux échantillons, du Khi-carré $\left(\chi^{2}\right)$ pour la comparaison des pourcentages (Schwartz, 1984), de l'Analyse des variances (ANOVA) et du logiciel Excel 2007.

\section{RESULTATS \\ Système de reproduction}

L'indice de fructification a été de 0,12 ( $\mathrm{s}=0,01), 0,09(\mathrm{~s}=0,03), 0,26(\mathrm{~s}=0,17)$ et $0,11(\mathrm{~s}=0,05)$ respectivement pour les traitements 1 et 2 en 2009 et en 2010. Ainsi en 2009, TC (Taux d'allogamie $)=24,83 \%$ et $T A$ (Taux d'autogamie) $=75,17 \%$, tandis qu'en 2010, $T C=56,42 \%$ et $T A$ (Taux d'autogamie) $=43,58 \%$. Pour les deux années cumulées, TC $=40,63 \%$ et TA $=59,38 \%$. Ainsi A. hypogaea a un régime de reproduction mixte allogameautogame, avec prédominance de l'autogamie.

\section{Diversité et abondance des insectes floricoles}

En 2009 et 2010 respectivement, 1446 et 1017 visites de 17 et 12 espèces d'insectes appartenant à 10 et 6 familles ont été dénombrées sur 36620 et 5760 fleurs de $A$. hypogaea. Le Tableau 1 présente la liste de ces insectes avec leur taux de visites.

D'après les données de ce tableau, les Hyménoptères constituent l'ordre le plus important avec 98,48\% des visites en 2009 et $91,25 \%$ en 2010. Ils sont principalement représentés par les Halictidés $(79,06 \%$ en 2009 et $25,95 \%$ en 2010), notamment Lasioglossum sp. (38,46\% en 2009 et $19,17 \%$ en 2010). Les plus intéressants des autres Hyménoptères sont les Apidés $(15,5 \%$ en 2009 et $58,01 \%$ en 2010), particulièrement 
Ceratina sp. $2(4,01 \%$ en 2009 et $23,89 \%$ en 2010). Les autres ordres sont plus rarement observés. Il s'agit des Coléoptères, des Lépidoptères et Diptères (moins de $7 \%$ des visites chacun, en 2009 comme en 2010).

Les conditions climatiques semblent quelquefois influencer l'activité de butinage des insectes sur les fleurs de A. hypogaea (Figure 2). En 2009, la corrélation s'est révélée positive et significative entre le nombre de visites des insectes et la température $(r=0,91 ; d d l=4 ; p<0,05)$, négative et significative entre l'humidité relative et le nombre des visites par tranche horaire $(r=-0,71 ; d d l=4 ; p<0,05)$. En 2010, la corrélation s'est révélée positive et non significative entre le nombre de visites des insectes et la température $(r=0,66 ; d d l=$ $4 ; p>0,05)$, négative et non significative entre le nombre des visites des insectes et l'humidité relative de l'air $(r=-0,51 ; d d l=4$; $p>0,05)$.

\section{Produits floraux récoltés}

Sur les fleurs de A. hypogaea, les insectes récoltent intensément et exclusivement du pollen (Figure 3 ). Toutes les 1531 et 1115 durées de visites enregistrées en 2009 et en 2010, étaient consacrées à la récolte du pollen.

Rythme des visites selon les étapes de la floraison

En général, les visites sont plus fréquentes sur les plantes lorsque le nombre de fleurs épanouies qu'elles portent est plus élevé. Nous avons trouvé une corrélation positive et hautement significative entre le nombre de fleurs épanouies et le nombre de visites d'insectes en 2009 ( $r=0,67 ; d d l=49$; $p<0,01)$ et en $2010(r=0,49 ; d d l=46 ; p<$ $0,01)$.

Rythme journalier des visites selon les étapes de la floraison

L'activité des insectes commence le matin avec l'épanouissement des fleurs et diminue fortement aux environs de $17 \mathrm{~h}$. La période journalière d'activité optimale des insectes se situe entre $10 \mathrm{~h}-11 \mathrm{~h}$ en 2009 et entre 8 h - 9 h en 2010 (Tableau 2).

\section{Abondance des insectes}

Le plus grand nombre d'individus simultanément en activité sur une fleur était de 1 en $2009(n=371, s=0)$ et en $2010(n=$ $180, \mathrm{~s}=0)$. Pour les abondances par 1000 fleurs, celles-ci variaient de 7,33 $(\mathrm{n}=60, \mathrm{~s}=$ 4,35) chez Ceratina sp. 2 à 19,02 ( $\mathrm{n}=52, \mathrm{~s}=$ 28,13) chez Halictus sp. en 2009 et de 6,54 (n $=51, \mathrm{~s}=6,31)$ chez Camponotus flavomarginatus à 21,13 $(\mathrm{n}=54, \mathrm{~s}=25,32)$ chez Ceratina sp. 1 en 2010 (Tableau 3).

\section{Durée des visites par fleur}

La durée moyenne d'une visite d'insecte par fleur de A. hypogaea variait de $25,94 \sec (\mathrm{n}=310 ; \mathrm{s}=18,68)$ chez Halictus sp. à $34,34 \sec (\mathrm{n}=505 ; \mathrm{s}=23,41)$ chez Ceratina sp. 1 en 2009 et de 10,53 sec ( $\mathrm{n}=$ $115 ; \mathrm{s}=9,84)$ chez Lasioglossum sp. 1 à $35,08 \sec (\mathrm{n}=316 ; \mathrm{s}=22,27)$ chez Ceratina sp. 1 en 2010 (Tableau 4).

\section{Vitesse de butinage}

La vitesse moyenne de butinage des fleurs de $A$. hypogaea variait de 1,96 fleurs/min $(\mathrm{n}=91 ; \mathrm{s}=1,61)$ chez Ceratina sp. 1 à 6 fleurs/min $(\mathrm{n}=83 ; \mathrm{s}=2,67) \mathrm{chez}$ Ceratina sp. 4 en 2009 et de 1,86 fleurs/min (n $=102 ; \mathrm{s}=1,60)$ chez Ceratina sp. 1 à 7,86 fleurs $/ \mathrm{min}(\mathrm{n}=76 ; \mathrm{s}=2,28) \mathrm{chez}$ Lasioglossum sp. 1 en 2010 (Tableau 5).

\section{Influence de la flore avoisinante}

Durant la période d'observation, plusieurs autres espèces végétales en fleurs en bordure de la parcelle de A. hypogaea étaient visitées pour leur nectar $(\mathrm{Ne})$ et/ou pour leur pollen (Po) par les insectes butineurs de cette Fabacée. Parmi ces plantes, nous citerons: Bidens pilosa (Asteraceae, $\mathrm{Ne}$ et Po), Brachiara brizantha (Fabaceae, Po), Cajanus cajan (Fabaceae, $\mathrm{Ne}$ et $\mathrm{Po}$ ), Callistemon rigidus (Myrtaceae, Ne et Po), Gossypium hirsutum (Malvaceae, Ne et Po), Phaseolus coccineus (Fabaceae, Ne), Phaseolus vulgaris (Fabaceae, Ne et Po), Senna mimosoides (Asteraceae, Ne et $\mathrm{Po}$ ), Tithonia diversifolia (Asteraceae, Ne et Po), Waltheria indica (Sterculiaceae, Ne et Po). Nous n'avons noté aucun passage d'insectes des fleurs de $A$. hypogaea aux fleurs d'autres espèces 
végétales et vice versa. Les butineuses étaient ainsi fidèles aux fleurs de A. hypogaea lors des voyages de butinage.

\section{Valeur apicole}

Pendant les périodes de floraison de $A$. hypogaea, nous avons noté une très faible activité des ouvrières de $A$. $m$. adansonii au niveau des fleurs de cette fabaceae. Seulement quatre visites ont été enregistrées et ceci en 2010, soit 0,39\% des visites d'insectes. Ces données mettent en évidence la faible attractivité du pollen de $A$. hypogaea vis-à-vis de A. m. adansonii. Elles permettent de classer cette espèce végétale parmi les plantes apicoles faiblement pollinifères.

Impact des insectes floricoles sur la pollinisation et les rendements en gousses et en graines

Pendant la récolte du pollen, la fréquence des visites avec contact entre les insectes et le stigmate est de $100 \%$ aussi bien en 2009 qu'en 2010. Ils augmentaient donc fortement les possibilités de pollinisation de A. hypogaea.

\section{Taux de fructification}

En 2009, le taux de fructification a été de $11,88 \%$ dans le traitement 1 et de $8,93 \%$ dans le traitement 2. La différence entre ces deux pourcentages est très hautement significative $\left(X^{2}=156,45 ; p<0,001\right)$. Le pourcentage $\left(P_{i}\right)$ du taux de fructification dû à l'influence des insectes floricoles a été de $24,83 \%$.

En 2010, le taux de fructification a été de $25,63 \%$ dans le traitement 1 et de $11,17 \%$ dans le traitement 2. La différence entre ces deux pourcentages est très hautement significative $\left(X^{2}=348,14 ; p<0,001\right)$. Le pourcentage $\left(P_{i}\right)$ du taux de fructification dû à l'influence des insectes floricoles a été de $54,47 \%$.

Pour l'ensemble des deux années d'études, le pourcentage $\left(P_{i}\right)$ du taux de fructification dû à l'influence des insectes floricoles a été de:

$P i=\{[(P i$ pour 2009) $+(P i$ pour 2010 $)] / 2\}=$ $39,65 \%$.

La différence entre les taux de fructification des traitements 1 est très hautement significative $\left(X^{2}=792,74 ; p<\right.$ $0,001)$. En conséquence, le nombre de gousses produit en 2010 a été plus élevé que celui de 2009.

L'influence des insectes anthophiles sur la fructification de A. hypogaea a donc été positive.

\section{Nombre moyen de graines par gousse}

En 2009, le nombre moyen de graines par gousse dans le traitement 1 a été de 1,93 $(\mathrm{n}=4351 ; \mathrm{s}=0,61)$ et de $1,64(\mathrm{n}=2805 ; \mathrm{s}=$ $0,6)$ dans le traitement 2. La différence entre ces moyennes est très hautement significative $(t=17,51 ; p<0,001)$. Le pourcentage $\left(P_{g}\right) \mathrm{du}$ nombre de graines par gousse attribuable à l'influence des insectes floricoles a été de $15,03 \%$.

En 2010, le nombre moyen de graines par gousse dans le traitement 1 a été de 2,08 $(\mathrm{n}=1476 ; \mathrm{s}=0,6)$ et de $1,77(\mathrm{n}=522 ; \mathrm{s}=$ $0,65)$ dans le traitement 2 . La différence entre ces moyennes est très hautement significative $(t=10,89 ; p<0,001)$. Le pourcentage $\left(P_{g}\right) \mathrm{du}$ nombre de graines par gousse attribuable à l'influence des insectes floricoles a été de $14,90 \%$.

Pour l'ensemble des deux années d'études, le nombre moyen de graines par gousse dû à l'influence des insectes floricoles a été de:

$P g=\{[(P g$ pour 2009) $+(P g$ pour 2010 $)] / 2\}$ $=14,97 \%$.

La différence entre le nombre moyen de graines par gousse des traitements 1 est très hautement significative $(t=7,13 ; p<0,001)$. En conséquence, le nombre de graines par gousse en 2010 a été plus élevé que celui de 2009.

L'influence des insectes anthophiles sur l'augmentation du nombre de graines par gousse de A. hypogaea a donc été positive.

\section{Pourcentage de graines normales}

En 2009, le pourcentage de graines normales a été de $91,68 \%$ dans le traitement 1 et de $65,41 \%$ dans le traitement 2. La différence entre ces deux pourcentages est très hautement significative $\left(X^{2}=1128,26 ; p<\right.$ $0,001)$. Le pourcentage des graines normales attribuable à l'influence des insectes floricoles a été de $28,65 \%$. 
En 2010, le pourcentage de graines normales a été de $92,73 \%$ dans le traitement 1 et de $69,65 \%$ dans le traitement 2. La différence entre ces deux pourcentages est très hautement significative $\left(X^{2}=246,64 ; p<\right.$ $0,001)$. Le pourcentage des graines normales attribuable à l'influence des insectes floricoles a été de 24,89\%.

Pour l'ensemble des deux années d'études, le pourcentage de graines normales dû à l'influence des insectes floricoles a été de:
$P n=\{[(P n$ pour 2009$)+(P n$ pour 2010$)] / 2\}$ $=26,77 \%$.

La différence entre le pourcentage de graines normales des traitements 1 est non significative $\left(X^{2}=2,53 ; p>0,05\right)$.

L'influence des insectes anthophiles sur la réduction du nombre de graines abortives par gousse de A. hypogaea a donc été positive.

Tableau 1 : Insectes recensés sur les fleurs de Arachis hypogaea en 2009 et 2010, nombre et pourcentage de visites des différents insectes.

\begin{tabular}{|c|c|c|c|c|c|c|c|c|}
\hline \multicolumn{3}{|l|}{ Insectes } & \multicolumn{2}{|c|}{2009} & \multicolumn{2}{|c|}{2010} & \multicolumn{2}{|c|}{ Total $_{2009 / 2010}$} \\
\hline Ordre & Famille & Genre, Espèce, Sous-espèce & $\mathbf{n}_{1}$ & $\begin{array}{c}\mathbf{P}_{1} \\
(\%)\end{array}$ & $\mathbf{n}_{2}$ & $\begin{array}{l}\mathbf{P}_{2} \\
(\%)\end{array}$ & $\mathbf{n}_{T}$ & $\begin{array}{l}\mathbf{P}_{\mathrm{T}} \\
(\%)\end{array}$ \\
\hline \multirow{17}{*}{ Hymenoptera } & \multirow{6}{*}{ Apidae } & $\begin{array}{l}\text { Apis mellifera adansonii } \\
\text { (pollen) }\end{array}$ & & & 4 & 0,39 & 4 & 0,16 \\
\hline & & Ceratina sp. 1 (pollen) & 124 & 8,58 & 37 & 3,64 & 161 & 6,54 \\
\hline & & Ceratina sp. 2 (pollen) & 58 & 4,01 & 243 & 23,89 & 301 & 12,22 \\
\hline & & Ceratina sp. 3 (pollen) & 12 & 0,83 & 206 & 20,26 & 218 & 8,85 \\
\hline & & Ceratina sp. 4 (pollen) & 30 & 2,08 & 100 & 9,83 & 130 & 5,28 \\
\hline & & Total Apidae & 224 & 15,5 & 590 & 58,01 & 814 & 33,05 \\
\hline & \multirow[t]{4}{*}{ Halictidae } & $\begin{array}{l}\text { Crocisaspidia chandleri } \\
\text { (pollen) }\end{array}$ & 65 & 4,5 & & & 65 & 2,64 \\
\hline & & Halictus sp. (pollen) & 535 & 36,1 & 69 & 6,78 & 604 & 24,52 \\
\hline & & Lasioglossum sp. 1 (pollen) & 556 & 38,46 & 195 & 19,17 & 751 & 30,49 \\
\hline & & Total Halictidae & 1156 & 79,06 & 264 & 25,95 & 1420 & $\mathbf{5 7 , 6 5}$ \\
\hline & \multirow[t]{4}{*}{ Formicidae } & $\begin{array}{l}\text { Camponotus flavomarginatus } \\
\text { (pollen) }\end{array}$ & . & , & 60 & 5,9 & 60 & 2,44 \\
\hline & & Camponotus sp. (pollen) & 26 & 1,8 & 14 & 1,38 & 40 & 1,62 \\
\hline & & Polyrachis sp. (pollen) & 10 & 0,69 & . & . & 10 & 0,41 \\
\hline & & Total Formicidae & 36 & 2,49 & 74 & 7,28 & 110 & 4,47 \\
\hline & Vespidae & $(1 \mathrm{sp}).($ pollen $)$ & 2 & 0,14 & . & . & 2 & 0,08 \\
\hline & Sphecidae & (1 sp.) (pollen) & 6 & 0,41 & - & & 6 & 0,24 \\
\hline & \multicolumn{2}{|c|}{ Total Hymenoptera } & 1424 & 98,48 & 928 & 91,25 & 2352 & 95,49 \\
\hline \multirow{4}{*}{ Coleoptera } & Meloidae & Coryna sp. (mange les fleurs) & 4 & 0,28 & 71 & 6,98 & 75 & 3,05 \\
\hline & Lagriidae & Lagria villosa (mange les & & & & & & \\
\hline & & fleurs) & 5 & 0,35 & . & · & 5 & 0,2 \\
\hline & \multicolumn{2}{|c|}{ Total Coleoptera } & 9 & 0,63 & 71 & 6,98 & 80 & 3,25 \\
\hline \multirow[t]{4}{*}{ Lepidoptera } & Pieridae & Eurema sp. 1 (pollen) & 3 & 0,21 & 9 & 0,88 & 12 & 0,49 \\
\hline & & Eurema sp. 2 (pollen) & 3 & 0,21 & . & (1) & 3 & 0,12 \\
\hline & Nymphalidae & Graphium pylades (pollen) & 3 & 0,21 & . & . & 3 & 0,12 \\
\hline & \multicolumn{2}{|c|}{ Total Lepidoptera } & 9 & $\mathbf{0 , 6 3}$ & 9 & $\mathbf{0 , 8 8}$ & 18 & $\mathbf{0 , 7 3}$ \\
\hline Diptera & Syrphidae & (1 sp.) (pollen) & 4 & 0,28 & 9 & 0,88 & 13 & 0,53 \\
\hline \multirow[b]{2}{*}{ Total } & & & 1446 & 100 & 1017 & 100 & 2463 & 100 \\
\hline & & & $\begin{array}{c}17 \\
\text { espèces }\end{array}$ & & $\begin{array}{c}12 \\
\text { espèces }\end{array}$ & & $\begin{array}{c}19 \\
\text { espèces }\end{array}$ & \\
\hline
\end{tabular}

$\mathrm{n}_{1}$ : nombre de visites sur 36620 fleurs en 52 jours, $\mathrm{n}_{2}$ : nombre de visites sur 5760 fleurs en 49 jours, $\mathrm{p}_{1}$ et $\mathrm{p}_{2}$ : pourcentages des visites, sp: espèces indéterminées. $\mathrm{p}_{1}=\left(\mathrm{n}_{1} / 1446\right) \times 100, \mathrm{p}_{2}=\left(\mathrm{n}_{2} / 1017\right) \times 100$. 
Tableau 2 : Fréquence des visites d'insectes sur les fleurs de Arachis hypogaea en fonction des tranches horaires d'observation en 2009 et 2010 à Dang.

\begin{tabular}{lccccccc}
\hline Année & Visites & \multicolumn{7}{c}{ Tranches horaires (heures) } \\
\cline { 3 - 8 } & & $\mathbf{6 - 7}$ & $\mathbf{8 - 9}$ & $\mathbf{1 0 - 1 1}$ & $\mathbf{1 2 - 1 3}$ & $\mathbf{1 4 - 1 5}$ & $\mathbf{1 6 - 1 7}$ \\
\hline 2009 & Nombre & 0 & 327 & 487 & 415 & 172 & 45 \\
& $\%$ & 0 & 22,61 & 33,68 & 28,7 & 11,89 & 3,11 \\
2010 & Nombre & 0 & 299 & 278 & 216 & 148 & 76 \\
& $\%$ & 0 & 29,4 & 27,34 & 21,24 & 14,55 & 7,47 \\
\hline
\end{tabular}

Tableau 3 : Abondance par 1000 fleurs de quelques insectes sur les fleurs de Arachis hypogaea en 2009 et 2010 à Dang.

\begin{tabular}{|c|c|c|c|c|c|c|c|}
\hline \multirow[t]{2}{*}{ Insectes } & \multirow[t]{2}{*}{ Années } & \multirow[t]{2}{*}{$\mathbf{n}$} & \multicolumn{5}{|c|}{ Abondance moyenne par 1000 fleurs (AMMF) } \\
\hline & & & $\mathbf{m}$ & $\mathbf{s}$ & mini & maxi & Comparaison des moyennes \\
\hline \multirow[t]{3}{*}{ Lasioglossum sp. 1} & 2009 & 52 & 7,41 & 22,75 & 12 & 152 & \multirow{3}{*}{$\mathrm{t}=-1,07 ; \mathrm{P}>0,05 ; \mathrm{NS}$} \\
\hline & 2010 & 85 & 10,91 & 7,77 & 3 & 60 & \\
\hline & $\mathrm{T}_{2009 / 2010}$ & 137 & 9,59 & 15,31 & 3 & 152 & \\
\hline Camponotus & 2009 & 52 & 12,68 & 24,82 & 12 & 152 & \multirow{3}{*}{$\mathrm{t}=1,73 ; \mathrm{P}>0,05 ; \mathrm{NS}$} \\
\hline \multirow[t]{2}{*}{ flavomarginatus } & 2010 & 51 & 6,54 & 6,31 & 3 & 60 & \\
\hline & $\mathrm{T}_{2009 / 2010}$ & 103 & 9,64 & 18,36 & 3 & 152 & \\
\hline \multirow[t]{3}{*}{ Ceratina sp. 1} & 2009 & 52 & 18,59 & 24,42 & 12 & 152 & \multirow{3}{*}{$\mathrm{t}=-0,53 ; \mathrm{P}>0,05 ; \mathrm{NS}$} \\
\hline & 2010 & 54 & 21,13 & 25,32 & 12 & 152 & \\
\hline & $\mathrm{T}_{2009 / 2010}$ & 106 & 19,87 & 24,80 & 12 & 152 & \\
\hline \multirow[t]{3}{*}{ Ceratina sp. 2} & 2009 & 60 & 7,33 & 4,35 & 5 & 71 & \multirow{3}{*}{$\mathrm{t}=-0,31 ; \mathrm{P}>0,05 ; \mathrm{NS}$} \\
\hline & 2010 & 61 & 7,58 & 4,62 & 5 & 59 & \\
\hline & $\mathrm{T}_{2009 / 2010}$ & 121 & 7,45 & 4,47 & 5 & 71 & \\
\hline \multirow[t]{3}{*}{ Ceratina sp. 3} & 2009 & 60 & 7,94 & 5,5 & 6 & 90 & \multirow{3}{*}{$\mathrm{t}=-0,45 ; \mathrm{P}>0,05 ; \mathrm{NS}$} \\
\hline & 2010 & 56 & 8,36 & 4,54 & 9 & 57 & \\
\hline & $\mathrm{T}_{2009 / 2010}$ & 116 & 8,14 & 5,04 & 6 & 90 & \\
\hline \multirow[t]{3}{*}{ Ceratina sp. 4} & 2009 & 58 & 9,96 & 6,80 & 4 & 90 & \multirow{3}{*}{$\mathrm{t}=-1,01 ; \mathrm{P}>0,05 ; \mathrm{NS}$} \\
\hline & 2010 & 64 & 11,25 & 7,26 & 4 & 70 & \\
\hline & $\mathrm{T}_{2009 / 2010}$ & 122 & 10,64 & 7,05 & 4 & 90 & \\
\hline \multirow[t]{3}{*}{ Coryna sp. } & 2009 & 66 & 7,98 & 3,06 & 5 & 77 & \multirow{3}{*}{$\mathrm{t}=-1,59 ; \mathrm{P}>0,05 ; \mathrm{NS}$} \\
\hline & 2010 & 61 & 8,96 & 3,82 & 5 & 59 & \\
\hline & $\mathrm{T}_{2009 / 2010}$ & 127 & 8,41 & 3,30 & 5 & 77 & \\
\hline \multirow[t]{3}{*}{ Eurema sp. 1} & 2009 & 64 & 8,72 & 4,3 & 5 & 74 & \multirow{3}{*}{$\mathrm{t}=-0,56 ; \mathrm{P}>0,05 ; \mathrm{NS}$} \\
\hline & 2010 & 53 & 9,17 & 4,38 & 5 & 54 & \\
\hline & $\mathrm{T}_{2009 / 2010}$ & 117 & 8,98 & 4,37 & 5 & 74 & \\
\hline \multirow[t]{3}{*}{ Halictus sp. } & 2009 & 52 & 19,02 & 28,13 & 12 & 152 & \multirow{3}{*}{$\mathrm{t}=-0,31 ; \mathrm{P}>0,05 ; \mathrm{NS}$} \\
\hline & 2010 & 51 & 20,80 & 29,68 & 12 & 147 & \\
\hline & $\mathrm{T}_{2009 / 2010}$ & 103 & 19,90 & 28,78 & 12 & 152 & \\
\hline Total & $\mathrm{T}_{2009 / 2010}$ & 1052 & 11,15 & 15,45 & 3 & 152 & $\mathrm{~F}=11,77 ; \mathrm{P}<0,05 ; \mathrm{DS}$ \\
\hline
\end{tabular}


Tableau 4: Durée des visites de quelques insectes par fleur de Arachis hypogaea à Dang en 2009 et 2010.

\begin{tabular}{|c|c|c|c|c|c|c|c|}
\hline \multirow[t]{2}{*}{ Insectes } & \multirow[t]{2}{*}{ Années } & \multirow[t]{2}{*}{$\mathbf{n}$} & \multicolumn{5}{|c|}{ Durée des visites par fleur (sec) } \\
\hline & & & m & $\mathbf{S}$ & mini & $\operatorname{maxi}$ & Comparaison des moyennes \\
\hline \multirow[t]{3}{*}{ Ceratina sp. 1} & 2009 & 506 & 34,34 & 23,41 & 2 & 98 & \\
\hline & 2010 & 316 & 35,08 & 22,27 & 2 & 97 & $t=-0,45 ; P>0,05 ; \mathrm{NS}$ \\
\hline & $\mathrm{T}_{2009 / 2010}$ & 822 & 34,63 & 22,97 & 2 & 98 & \\
\hline \multirow[t]{3}{*}{ Ceratina sp. 2} & 2009 & 153 & 32,44 & 23,98 & 2 & 109 & \\
\hline & 2010 & 181 & 29,58 & 25,29 & 2 & 109 & $t=1,06 ; P>0,05 ; \mathrm{NS}$ \\
\hline & $\mathrm{T}_{2009 / 2010}$ & 334 & 30,89 & 24,70 & 2 & 109 & \\
\hline \multirow[t]{3}{*}{ Ceratina sp. 3} & 2009 & 173 & 31,16 & 22,31 & 2 & 107 & \\
\hline & 2010 & 162 & 30,34 & 20,15 & 2 & 87 & $t=0,35 ; P>0,05 ; \mathrm{NS}$ \\
\hline & $\mathrm{T}_{2009 / 2010}$ & 335 & 29,99 & 21,30 & 2 & 107 & \\
\hline \multirow[t]{3}{*}{ Ceratina sp. 4} & 2009 & 87 & 30,44 & 20,15 & 2 & 90 & \\
\hline & 2010 & 95 & 32,46 & 21,96 & 3 & 104 & $t=-0,65 ; P>0,05 ; \mathrm{NS}$ \\
\hline & $\mathrm{T}_{2009 / 2010}$ & 182 & 30,89 & 20,17 & 2 & 104 & \\
\hline \multirow[t]{3}{*}{ Halictus sp. } & 2009 & 310 & 25,94 & 18,68 & 2 & 87 & \\
\hline & 2010 & 170 & 13,84 & 12,27 & 2 & 52 & $t=8,53 ; P<0,001 ;$ THS \\
\hline & $\mathrm{T}_{2009 / 2010}$ & 480 & 21,82 & 17,67 & 2 & 87 & \\
\hline \multirow[t]{3}{*}{ Crocisaspidia chandleri } & 2009 & 96 & 28,2 & 18,28 & 2 & 83 & \\
\hline & 2010 & 77 & 27,69 & 17,82 & 2 & 83 & $t=0,18 ; P>0,05 ; \mathrm{NS}$ \\
\hline & $\mathrm{T}_{2009 / 2010}$ & 173 & 27,97 & 18,03 & 2 & 83 & \\
\hline \multirow[t]{3}{*}{ Lasioglossum sp. 1} & 2009 & 206 & 30,73 & 20,52 & 2 & 91 & \\
\hline & 2010 & 114 & 10,53 & 9,84 & 1 & 63 & $t=11,89 ; P<0,001 ; \mathrm{THS}$ \\
\hline & $\mathrm{T}_{2009 / 2010}$ & 320 & 23,47 & 19,95 & 1 & 91 & \\
\hline Total & $\mathrm{T}_{2009 / 2010}$ & 2646 & 30,47 & 22,23 & 1 & 109 & $F=13,30 ; P<0,05 ; \mathrm{DS}$ \\
\hline
\end{tabular}

Tableau 5 : Vitesse de butinage de quelques insectes sur les fleurs de Arachis hypogaea à Dang en 2009 et 2010 .

\begin{tabular}{|c|c|c|c|c|c|c|c|}
\hline \multirow[t]{2}{*}{ Insectes } & \multirow[t]{2}{*}{ Années } & \multirow[t]{2}{*}{$\mathbf{n}$} & \multicolumn{5}{|c|}{ Vitesse de butinage (min) } \\
\hline & & & $\mathbf{m}$ & $\mathbf{s}$ & mini & $\operatorname{maxi}$ & Comparaison des moyennes \\
\hline \multirow[t]{3}{*}{ Ceratina sp. 1} & 2009 & 91 & 1,96 & 1,61 & 0,45 & 4,76 & \\
\hline & 2010 & 102 & 1,86 & 1,60 & 0,45 & 4,76 & $t=0,43 ; P>0,05 ; \mathrm{NS}$ \\
\hline & $\mathrm{T}_{2009 / 2010}$ & 193 & 1,89 & 1,57 & 0,45 & 4,76 & \\
\hline \multirow[t]{3}{*}{ Ceratina sp. 2} & 2009 & 99 & 2,82 & 1,68 & 0,85 & 7,5 & \\
\hline & 2010 & 115 & 2,95 & 1,92 & 0,85 & 8,57 & $t=-0,53 ; P>0,05 ; \mathrm{NS}$ \\
\hline & $\mathrm{T}_{2009 / 2010}$ & 214 & 2,89 & 1,81 & 0,85 & 8,57 & \\
\hline \multirow[t]{3}{*}{ Ceratina sp. 3} & 2009 & 100 & 2,38 & 1,63 & 1,31 & 2,1 & \\
\hline & 2010 & 129 & 2,44 & 1,64 & 1,04 & 8,57 & $t=-0,28 ; P>0,05 ; \mathrm{NS}$ \\
\hline & $\mathrm{T}_{2009 / 2010}$ & 229 & 2,42 & 1,63 & 1,04 & 8,57 & \\
\hline \multirow[t]{3}{*}{ Ceratina sp. 4} & 2009 & 83 & 6,00 & 2,67 & 1,98 & 13,85 & \\
\hline & 2010 & 61 & 6,78 & 3,17 & 1,98 & 13,9 & $t=-1,56 ; P>0,05 ; \mathrm{NS}$ \\
\hline & $\mathrm{T}_{2009 / 2010}$ & 144 & 6,42 & 3,22 & 1,98 & 13,85 & \\
\hline \multirow[t]{3}{*}{ Halictus sp. } & 2009 & 63 & 2,18 & 1,95 & 0,41 & 11,76 & \\
\hline & 2010 & 51 & 1,91 & 1,65 & 0,41 & 10,00 & $t=0,80 ; P>0,05 ; \mathrm{NS}$ \\
\hline & $\mathrm{T}_{2009 / 2010}$ & 114 & 2,06 & 1,82 & 0,41 & 11,76 & \\
\hline Lasioglossum sp. & 2009 & 44 & 2,93 & 2,68 & 0,56 & 15,00 & \\
\hline \multirow{2}{*}{1} & 2010 & 76 & 7,86 & 2,28 & 3,87 & 13,30 & $t=-10,24 ; P<0,001 ;$ THS \\
\hline & $\mathrm{T}_{2009 / 2010}$ & 120 & 6,05 & 3,41 & 0,56 & 15,00 & \\
\hline Total & $\mathrm{T}_{2009 / 2010}$ & 1014 & 3,38 & 2,83 & 0,41 & 15,00 & $F=123,70 ; P<0,05 ; \mathrm{DS}$ \\
\hline
\end{tabular}


Tableau 6 : Taux de fructification, nombre moyen de graines par gousse et pourcentage des graines normales selon les traitements de Arachis hypogaea en 2009 et 2010 à Dang.

\begin{tabular}{|c|c|c|c|c|c|c|c|c|c|c|}
\hline \multirow[t]{2}{*}{ Années } & \multirow[t]{2}{*}{ Traitements } & \multirow[t]{2}{*}{ NFE } & \multirow[t]{2}{*}{$\mathrm{NgF}$} & \multirow{2}{*}{$\begin{array}{c}\text { TF } \\
(\%)\end{array}$} & \multicolumn{3}{|c|}{ Graines/gousse } & \multirow[b]{2}{*}{ NTG } & \multirow[b]{2}{*}{ NGN } & \multirow{2}{*}{$\begin{array}{l}\% \\
\text { GN }\end{array}$} \\
\hline & & & & & $\mathbf{n}$ & $\mathbf{m}$ & $\mathbf{S}$ & & & \\
\hline \multirow[t]{2}{*}{2009} & $1(\mathrm{Fl})$ & 36620 & 4351 & 11,88 & 4351 & 1,93 & 0,61 & 6921 & 6345 & 91,68 \\
\hline & $2(\mathrm{Fi})$ & 31410 & 2805 & 8,93 & 2805 & 1,64 & 0,6 & 3510 & 2297 & 65,41 \\
\hline \multirow[t]{2}{*}{2010} & 1 (Fl) & 5760 & 1476 & 25,63 & 1476 & 2,08 & 0,6 & 2255 & 2091 & 92,73 \\
\hline & $2(\mathrm{Fi})$ & 4673 & 522 & 11,17 & 522 & 1,77 & 0,65 & 649 & 452 & 69,65 \\
\hline
\end{tabular}

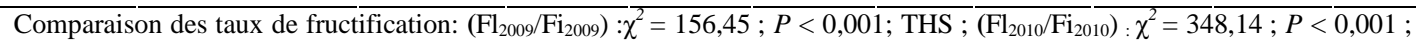
THS $;\left(\mathrm{Fl}_{2009} / \mathrm{Fl}_{2010}\right): \chi^{2}=792,74 ; P<0,001 ;$ THS $; \chi_{\text {Global }}^{2}=654,01 ; P<0,001 ;$ THS, Comparaison des nombres moyens de graines/gousse: $\left(\mathrm{Fl}_{2009} / \mathrm{Fi}_{2009):} t=17,51 ; P<0,001 ; \mathrm{THS} ;\left(\mathrm{Fl}_{2010} / \mathrm{Fi}_{2010}\right): t=10,89 ; P<0,001 ; \mathrm{THS} ;\left(\mathrm{Fl}_{2009} / \mathrm{Fl}_{2010}\right): t=7,13\right.$; $P<0,001$; THS, Comparaison des pourcentages de graines normales: $\left(\mathrm{Fl}_{2009} / \mathrm{Fi}_{2009}\right): \chi^{2}=1128,26 ; P<0,001$; THS ; $\left(\mathrm{Fl}_{2010} / \mathrm{Fi}_{2010}\right): \chi^{2}=246,64 ; P<0,001$; THS $;\left(\mathrm{Fl}_{2009} / \mathrm{Fl}_{2010}\right): \chi^{2}=2,53 ; P>0,05 ; \mathrm{NS} ; \chi_{\text {Global }}^{2}=37,42 ; P<0,001$; THS, Comparaison globale des moyennes: $F=372,16 ; P<0,05$; DS.

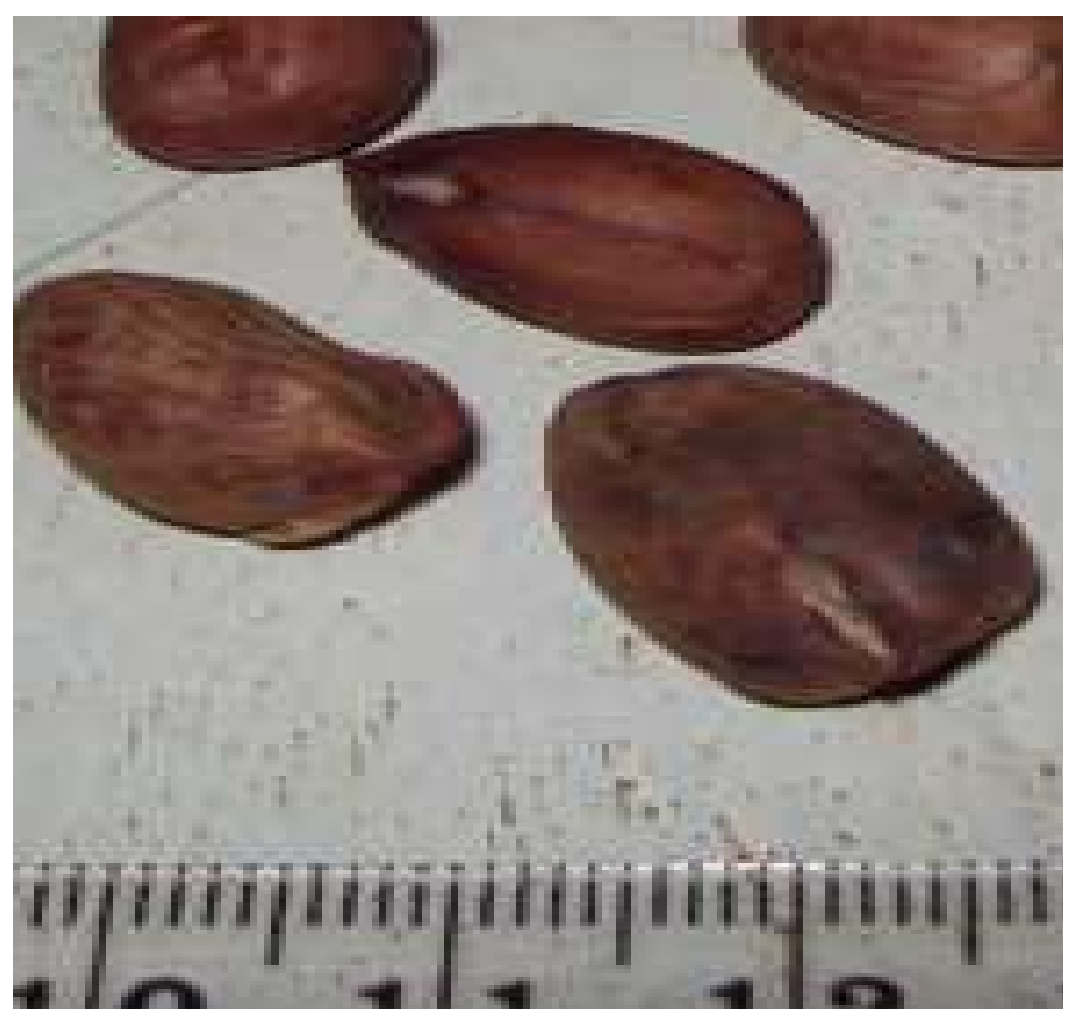

Figure 1 : Graines de Arachis hypogaea de la variété 55-437 RMP-91. 

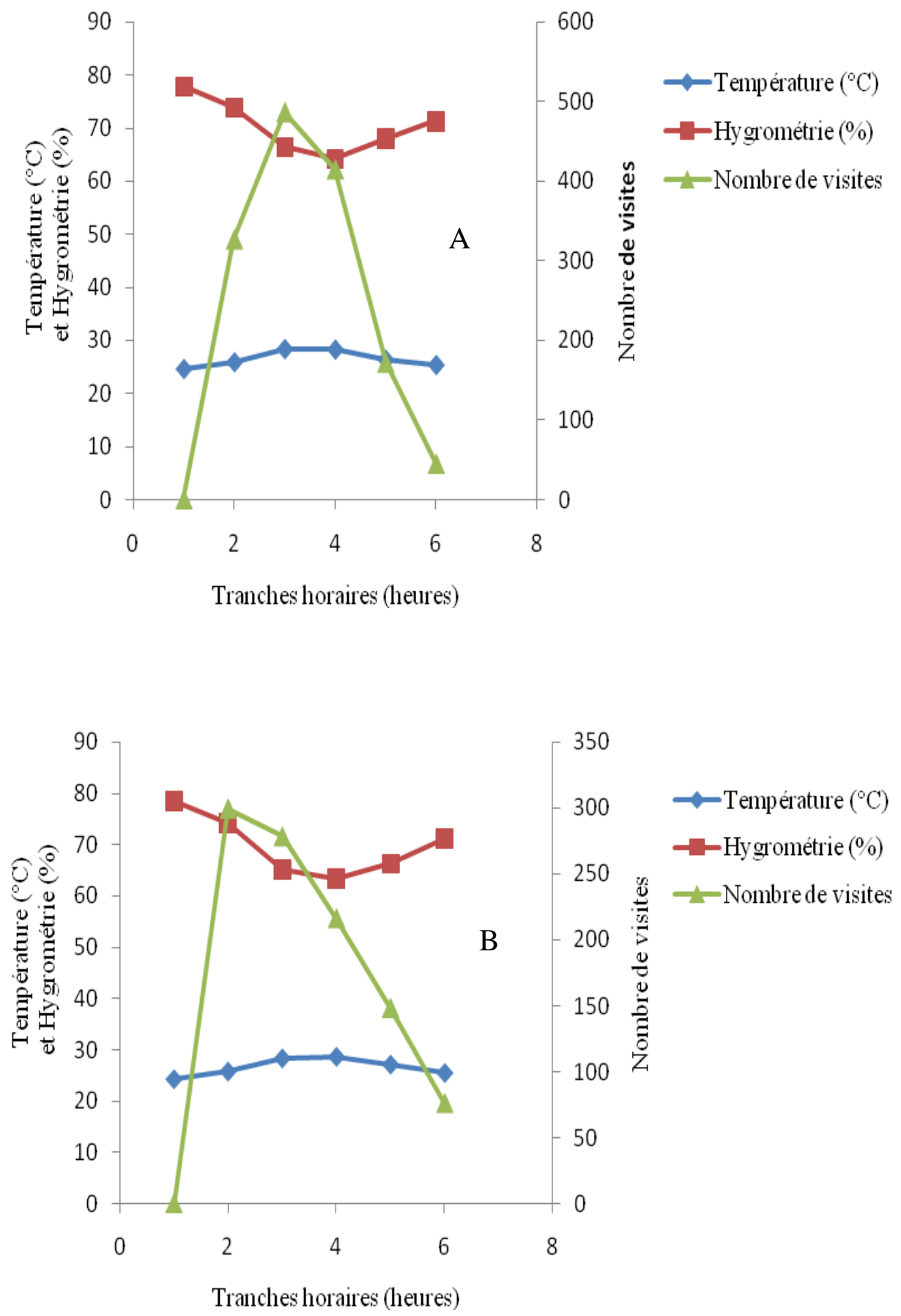

Figure 2 : Distribution journalière du nombre des visites d'insectes sur 36620 et 5760 fleurs de Arachis hypogaea en 2009 (A) et 2010 (B) respectivement, en fonction de la température et de l'hygrométrie moyenne. 


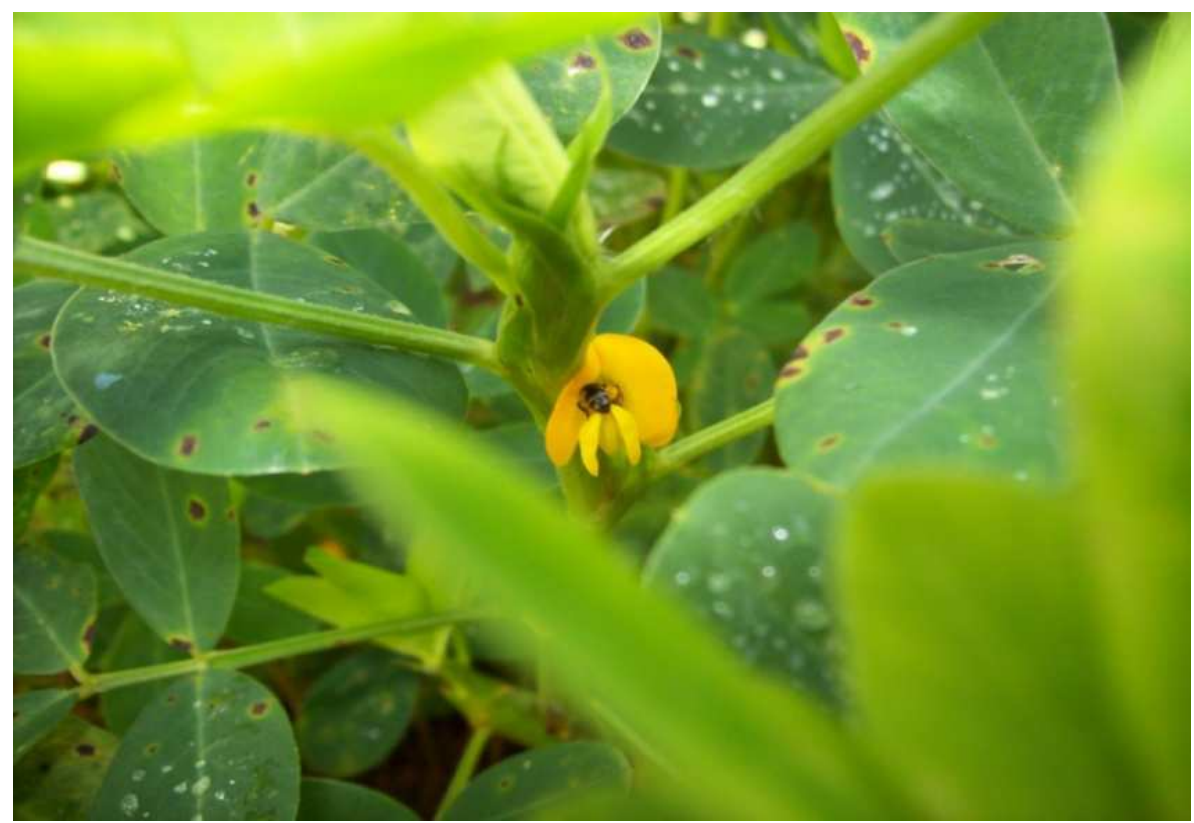

Figure 3 : Ceratina sp. récoltant du pollen dans une fleur de Arachis hypogaea.

\section{DISCUSSION}

Activité des insectes sur les fleurs de Arachis hypogaea

A Dang (dans la région de l'Adamaoua), les principaux insectes anthophiles de A. hypogaea appartiennent à l'ordre des Hyménoptères, avec la famille des Halictidae qui est la plus abondante. Ces résultats sont similaires à ceux obtenus par Tchuenguem Fohouo (1993) qui a travaillé sur l'activité des insectes anthophiles de l'arachide dans la zone de Nkolbisson (Yaoundé) et a trouvé que l'ordre le plus important est celui des Hyménoptères (93,56\% des visites) avec la famille des Halictidae qui est la plus abondante. La diversité et l'abondance des insectes anthophiles de A. hypogaea peuvent varier avec les régions. Ainsi, à Java, Heide (1923) a mentionné que les Lycaenidae, les Xylocopinés, les Ceratinidés et les Apidés (notamment Melipona sp. et Apis cerana) étaient les plus abondants. De même, en Israël, Carmin (Free, 1970) a noté que Ceratina bispinosa est le principal insecte visiteur. Par contre, au Malawi, Apis mellifica, Nomia spp. et Megachile spp. ont été les visiteurs les plus communs (Gibbons et Tattersfield, 1969).

Dans l'ensemble, les insectes sont abondants sur les fleurs dans la matinée. Cette période journalière correspondrait au moment de la journée où le pollen de A. hypogaea est disponible en grande quantité. Ces observations corroborent celles de Hammons et al. (1963) qui indiquent que les Halictidae et les Megachilidae étaient très abondants sur les plantes dans la matinée, au moment où le pollen est libéré.

La faible abondance des butineuses par fleur et par 1000 fleurs serait liée à l'absence de nectar dans la fleur et à la faible production de pollen par la plante. Les mêmes résultats ont été rapportés à Nkolbisson par Tchuenguem Fohouo (1993). Par contre, à Campo, Tchuenguem Fohouo et al. (2000) ont trouvé 4 ouvrières de Apis mellifica sur un pied de A. hypogaea.

La durée moyenne d'une visite de récolte de pollen varie avec l'insecte et ceci d'une année à une autre. La durée des visites semble être liée à l'accessibilité au pollen de A. hypogaea. Nos données confirment celles de Leuk et Hammons (1965) qui ont indiqué 
que la durée d'une visite d'Halictidé peut dépasser 180 secondes par fleur. Ceci pourrait s'expliquer par des interruptions de visites d'insectes chercheurs de pollen par d'autres insectes. L'interruption d'une visite a lieu à la suite des pluies violentes, des collisions entre les visiteurs, des tentatives de capture du visiteur par un prédateur ou de l'approche de la fleur déjà occupée par un premier visiteur. L'interruption des visites chez les Apoïdes peut entraîner la réduction de la durée d'une visite par fleur et la perte d'une partie du pollen transporté. Ceci a pour conséquence la prolongation de l'activité exploratrice de l'Apoïde lors d'un voyage de butinage.

Pour l'ensemble des insectes butineurs, le passage de A. hypogaea à une autre plante avoisinante n'a pas été observé. Ceci prouve que les butineuses des fleurs de A. hypogaea étaient fidèles à cette Fabaceae. Ce phénomène est connu sous l'expression «constance florale» (Basualdo et al., 2000). La constance florale est un aspect important dans la gestion de la pollinisation.

Nous avons noté une très faible activité des ouvrières de A. m. adansonii sur les fleurs de A. hypogaea durant nos investigations. Ceci serait dû au fait que le pollen de cette plante n'est pas attractif pour cette abeille dans nos conditions d'observation.. La valeur apicole de cette fabaceae est presque nulle à Dang.

\section{Impact de l'activité des insectes sur la pollinisation et les rendements de Arachis hypogaea}

$\mathrm{Au}$ cours de leur récolte de pollen, les insectes rentrent fréquemment en contact avec le stigmate. Ils peuvent intervenir directement dans l'autopollinisation, en mettant le pollen d'une fleur sur le stigmate de celle-ci. Ceci est d'autant plus probable que l'autogamie est prépondérante chez A. hypogaea (Hammons et Leuk, 1966).

Les insectes (principalement les Apoïdes) transportent le pollen de fleur en fleur et peuvent ainsi jouer un rôle positif dans la geitogamie, en mettant le pollen d'une fleur sur le stigmate d'une autre fleur de la même plante. Les insectes qui passent de fleur en fleur sur différentes plantes peuvent transporter le pollen d'une plante à une autre.
Ils peuvent favoriser la xénogamie, en mettant le pollen d'une plante sur le stigmate d'une fleur d'une autre plante. Cette dernière forme de pollinisation est d'autant plus probable que l'allogamie existe chez A. hypogaea (Hammons et Leuk, 1966).

Les insectes secouent les fleurs pendant leurs visites. Ce mouvement faciliterait la libération optimale de pollen pour l'occupation du stigmate. La charge optimale de pollen sur le stigmate serait favorable à la formation des gousses et des graines. En effet, Segeren et al. (1996) ont montré qu'une pollinisation efficace permet l'augmentation des rendements et la qualité du fruit est meilleure. En agitant les fleurs, les insectes anthophiles interviennent dans l'autopollinisation. Comme l'avait signalé Tchuenguem Fohouo (1993), le rôle des insectes sur l'autopollinisation de l'arachide serait proportionnel à l'aptitude à provoquer l'agitation de la fleur. Pour cette raison, il est attribuable principalement aux Apoïdes. Girardeau et Leuck (1967) ont d'ailleurs montré que les fleurs d'arachide encagées et non manipulées produisent moins de gousses (de 4 à 10\%) que les fleurs également encagées, mais constamment agitées à la main.

Le taux d'intervention des insectes floricoles sur la productivité de l'arachide est connu dans d'autres régions : en Egypte, les insectes ont augmenté la productivité grainière d'environ 46\% (Rashad et al., 1978). Girardeau et Leuk (1967) ont signalé qu'en Georgie, les Apoïdes augmentent les rendements en gousses de 6 à $11 \%$.

\section{Conclusion}

A Ngaoundéré, A. hypogaea est une plante pollinifère qui bénéficie de la pollinisation par les insectes. En comparant le rendement des plantes non protégées à celui des plantes protégées des insectes, il est apparu que les insectes anthophiles ont augmenté le taux de fructification, le nombre moyen de graines par gousse ainsi que le pourcentage de graines normales en 2009 et 2010. La grande majorité des insectes inventoriés sur les fleurs d'arachide sont des pollinisateurs de cette plante. Les plus intéressants d'entre eux sont des Abeilles de la 
famille des Halictidés qui sont de bons récolteurs du pollen de la Fabacée. Le traitement des plants d'arachide aux pesticides chimiques est à éviter pendant la période de floraison, afin de ne pas menacer les insectes pollinisateurs.

\section{REFERENCES}

Basualdo M, Bedascarrasbure E, De Jong D. 2000. Africanized honey bees (Hymenoptera: Apidae) have a greater fidelity to sunflowers than European bees. J. Econ. Entomol., 2: 304-307.

Demarly. 1977. Génétique et Amélioration des Plantes. Masson: Paris; 577.

Dongock ND, Foko J, Pinta LV, Tchoumboue J, Zango P. 2004. Inventaire et identification des plantes mellifères de la zone soudano-guinéenne d'altitude de l'Ouest Cameroun. Tropicultura, 22(3): 139-145.

Douka C, Tchuenguem Fohouo F-N. 2013 Foraging and pollination behavior of Apis mellifera adansonii L. (Hymenoptera, Apidae) on Phaseolus vulgaris (Fabaceae) flowers at Maroua (Cameroon). International Research Journal of Plant Science, 4(2): 45-54.

Dounia, Tchuenguem Fohouo F-N. 2013. Foraging and pollination activity of Apis mellifera adansonii Latreille (Hymenoptera: Apidae) on flowers of Gossypium hirsutum L. (Malvaceae) at Maroua, Cameroon. International Research Journal of Plant Science, 4(2): $33-44$.

DSCE. 2009. Document de stratégie pour la croissance et l'emploi. MINEPAT, Cameroun, 112.

Fameni TS, Tchuenguem Fohouo F-N, Brückner D. 2012. Pollination efficiency of Apis mellifera adansonii Latreille (Hymenoptera, Apidae) on Callistemon rigidus $\mathrm{R}$. Br. 1819 flowers at Dang (Ngaoundere-Cameroon). IJTIS. 32(1): 211.

Free JB. 1970. Insect Pollinisation of Crops. Academic Press: London, New York; 544.

FAO. 2003. Commission du codex alimentarius. Rapport de la dix-huitième session du comité du codex sur les graisses et les huiles, Londres, 78.

Gibbons RW, Tattersfield JR. 1969. Outcrossing trials with groundnuts (Arachis hypogaea L.). Rhodesia Zambia Malawi Journ. Agr. Res., 7: 71-75.

Girardeau JH, Leuk DB. 1967. Effect of mechanical and bee tripping on yield of the peanut. Journ. Econ. Entomol., 60: 1454-1455.

Heide FFR. 1923. Biological observations of Arachis hypogaea L. Alg. Proefsta. V. Landb. Meded., 14: 1-19.

Hammons RO, Leuk DB. 1966. Natural crosspollination of the peanut, Arachis hypogaea L., in the presence of bees and thrips. Agronomy Journal, 58: 396.

Hammons RO, Krombein KV, Leuk DB. 1963. Somme bees (Apoidea) associated with peanut flowering. Journ. Econ. Entomol., 56: 905.

Jean-Prost P. 1987. Apiculture : Connaître l'Abeille - Conduire le Rucher (6 $6^{\mathrm{è}}$ éd), Lavoisier: Paris; 579.

Jacob-Remacle A. 1989. Comportement de butinage de l'abeille domestique et des Abeilles sauvages dans des vergers de pommiers en Belgique. Apidologie, 20(4): 271-285.

Kalfaoui JLB. 1988. Approche de l'amélioration génétique de l'adaptation à la sécheresse des espèces cultivées en zone semi-aride. Application au cas de l'arachide (Arachis hypogaea L.) destinée à la région sèche du Sénégal. Thèse de Doctorat, Université Paris-Sud, p. 67-68.

Kingha TBM, Tchuenguem Fohouo F-N, Ngakou A, Brückner D. 2012. Foraging and pollination activities of Xylocopa olivacea (Hymenoptera, Apidae) on Phaseolus vulgaris (Fabaceae) flowers at Dang (Ngaoundere - Cameroon). J.A.E.R.D., 4(6): 330-339.

Leuk DB, Hammons RO. 1965. Pollen collecting activities of bees among peanut flowers. Journ. Econ. Entomol., 58: 10281030 .

MINADER/DESA. 2010. Annuaire des Statistiques du Secteur Agricole Campagnes $2007 \&$ 2008. AGRI-STAT, 16: 98. 
Pando JB, Tchuenguem Fohouo F-N, Tamesse JL. 2011a. Foraging and pollination behaviour of Xylocopa calens Lepeletier (Hymenoptera: Apidae) on Phaseolus coccineus L. (Fabaceae) flowers at Yaoundé (Cameroon). Entomol. Res., 41: 185-193.

Pando JB, Tchuenguem Fohouo F-N, Tamesse JL. 2011b. Pollination and yield responses of pigeon pea (Cajanus cajan L. Mill sp.) to the foraging activity of Chalicodoma cincta cincta (Hymenoptera: Megachilidae) in Yaoundé (Cameroon). J.A.P.S., 11(1): 1346-1357.

Pouvreau A. 1984. Cultures tropicales oléagineuses. In Pollinisation et Productions végétales. PESSON P., LOUVEAUX J., INRA: Paris; 331-347.

Praquin JY, Tardieu M. 1976. L'arachide dans les zones d'altitude de l'ouest-cameroun. L'agronomie Tropicale, 31(1): 83-89.

Rashad SE, Ewies MA, El Rabie HG. 1978. Pollinators of peanuts (Arachis hypogaea L.) and the effect of honeybees on its yield. In $4^{\text {th }}$ intern. Simp. on pollination. Md. Agric. Exp. Sta. Spec. Misc. Publ., 1: 227-230.

Roubik DW. 2000. Pollination system stability in Tropical America. Conserv. Biol., 14(5): 1235-1236.

Schwartz. 1984. Méthodes Statistiques à l'Usage des Médecins et des Biologistes. Flammarion Médecine-Science; Paris; 318.

Segeren P, Mulder V, Beetsma J, Sommeijer R. 1996. Apiculture sous les tropiques. Agrodok 32, ( $5^{\text {ème }}$ edn), Agromisa, Wageningen; 88.

Tchuenguem Fohouo F-N. 1993. Activités des insectes anthophiles et son impact sur les rendements de deux plantes cultivées au Cameroun : Zea mays L. (Gramineae) et Arachis hypogaea L. (Papilionaceae). Thèse de Doctorat de 3è Cycle, Université de Yaoundé I, p.133.

Tchuenguem Fohouo F-N, 2005. Activité de butinage et de pollinisation d'Apis mellifera adansonii Latreille (Hymenoptera: Apidae, Apinae) sur les fleurs de trois plantes à Ngaoundéré (Cameroun): Callistemon rigidus (Myrtaceae), Syzygium guineense var. macrocarpum (Myrtaceae) et Voacanga africana (Apocynaceae). Thèse de Doctorat d'Etat, Université de Yaoundé I, p. 103.

Tchuenguem Fohouo F-N, Messi J, Nkongmeneck BA. 2000. Exploitation des fleurs de quelques plantes par les insectes à Campo (Sud-Cameroun). Rev. Sci. Technol. Dev., 1(7): 12-17.

Tchuenguem Fohouo F-N, Messi J, Pauly A. 2001. Activité de Meliponula erythra sur les fleurs de Dacryodes edulis et son impact sur la fructification. Fruits, 56: 179-188.

Tchuenguem Fohouo F-N, Djonwangwe D, Messi J, Brückner D. 2007. Exploitation of Entada africana, Eucalyptus camaldulensis, Psidium guajava and Trichillia emetica flowers by Apis mellifera adansonii at Dang (Ngaoundéré, Cameroon). Cameroon Journal of Experimental Biology, 3: 50-60.

Tchuenguem Fohouo F-N, Djonwangwe D, Messi J, Brückner D. 2009a. Activité de butinage et de pollinisation d'Apis mellifera adansonii sur les fleurs de Helianthus annus (Asteraceae) à Ngaoundéré (Cameroun). Cameroon Journal of Experimental Biology, 5: 1-9.

Tchuenguem Fohouo F-N, Ngakou A, Kengni BS. 2009b. Pollination and yield responses of cowpea (Vigna unguiculata L. Walp.) to the foraging activity of Apis mellifera adansonii (Hymenoptera: Apidae) at Ngaoundéré (Cameroon). African Journal of Biotechnology, 9: 1988-1996.

Vallardir F. 1964. Encyclopédie du Monde végétal. Tome II. Lidis Edition, Paris; 532. 